\title{
A Novel Homozygous CAPN1 Pathogenic Variant in a Chinese Patient with Pure Hereditary Spastic Paraplegia
}

\author{
You Chen ${ }^{\mathrm{a}}$

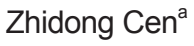 \\ Xiaosheng Zheng ${ }^{\mathrm{a}, \mathrm{b}}$ \\ Fei Xie $\mathrm{a}^{\mathrm{a}, \mathrm{c}}$ \\ Si Chen ${ }^{\mathrm{a}, \mathrm{d}}$ \\ Wei Luo ${ }^{\mathrm{a}}$ \\ aDepartment of Neurology, \\ Second Affiliated Hospital, \\ Zhejiang University School of Medicine, \\ Hangzhou, China \\ 'Intensive Care Unit, Zhejiang Hospital, \\ Hangzhou, China \\ 'Department of Neurology, \\ Sir Run Run Shaw Hospital, \\ Zhejiang University School of Medicine, \\ Hangzhou, China \\ ${ }^{\mathrm{d}}$ Cancer Institute, Key Laboratory \\ of Cancer Prevention and Intervention, \\ China National Ministry of Education, \\ Second Affiliated Hospital, \\ Zhejiang University School of Medicine, \\ Hangzhou, China
}

Dear Editor,

Hereditary spastic paraplegias (HSPs) are clinically and genetically heterogeneous neurological disorders characterized by lower limb spasticity and weakness, with or without additional neurological symptoms or other system involvement, which are defined as complicated and pure phenotypes, respectively. ${ }^{1}$ CAPN1 (MIM: 114220) has recently been identified as the causative gene for spastic paraplegia 76 (SPG76), an autosomal recessive HSP associated with either a pure or complicated phenotype, accompanied by ataxia, mild cognitive decline, or axonal peripheral involvement. ${ }^{2,3}$ Here we report the first Chinese HSP patient with biallelic CAPN1 pathogenic variants.

Our 38-year-old female patient (Fig. 1A), born into a consanguineous Chinese family, first noticed symptoms of slowly progressive lower limb stiffness and unsteadiness at the age of 35 years. No bladder dysfunction was reported. A neurological examination showed symmetrical hyperreflexia in both the upper and lower extremities. The muscle tension was increased in all four extremities, she was positive for bilateral patellar clonus, and bilateral pes cavus was observed. The patient did not present signs of cerebellar ataxia. The muscle force was normal in all limbs. Hematological and biochemical tests, a nerve electrophysiological study, and brain MRI all produced unremarkable findings. Her healthy parents and younger sister were also comprehensively checked by two experienced neurologists.

The homozygosity mapping revealed 19 extended (>2.0 Mb) runs of homozygosity harboring 7 candidate genes responsible for autosomal recessive HSPs. Direct sequencing of these candidate genes identified a novel homozygous nonsense variant (c.1015C $>\mathrm{T}$ ) in CAPN1 (NM_001198868), resulting in a premature stop codon (p.R339*) (Fig. 1B). Cosegregation analysis revealed that the pathogenic variant was cosegregated with the disease in the family. The healthy parents of the patient and her younger sister were all heterozygotes for the c. $1015 \mathrm{C}>\mathrm{T}$ variant (Fig. 1B).

CAPN1 has both pure and complicated phenotypes, and our patient presented with a pure phenotype. Ataxia is the most prominent feature in patients with a complicated phenotype. CAPN1 encodes the protein calpain-1, with a high expression level in the cerebellum during the prenatal and early postnatal periods, indicating a potential role for calpain-1 in cerebellar development. ${ }^{4}$ It is particularly interesting that a missense CAPN1 variant was found to be associated with spinocerebellar ataxia in the Parson Russell Terrier dog breed. ${ }^{5}$ Calpain-1 homozygous knockout mice also exhibit a mild form of ataxia due to abnormal cerebellar development, including altered synaptic transmission, enhanced neuronal apoptosis, and decreased number of cerebellar granule cells. ${ }^{3}$

In conclusion, we report a Chinese patient with a pure HSP phenotype caused by a novel nonsense homozygous variant (c.1015C>T, p.R339*) in CAPN1. This is the first report of a CAPN1 pathogenic variant in a patient of Chinese descent. Additional studies are needed to evaluate the frequency and phenotype-genotype correlation of CAPN1 in the Chinese

(a) This is an Open Access article distributed under the terms of the Creative Commons Attribution Non-Commercial License (https://creativecommons.org/licenses/by-nc/4.0) which permits unrestricted non-commercial use, distribution, and reproduction in any medium, provided the original work is properly cited. 


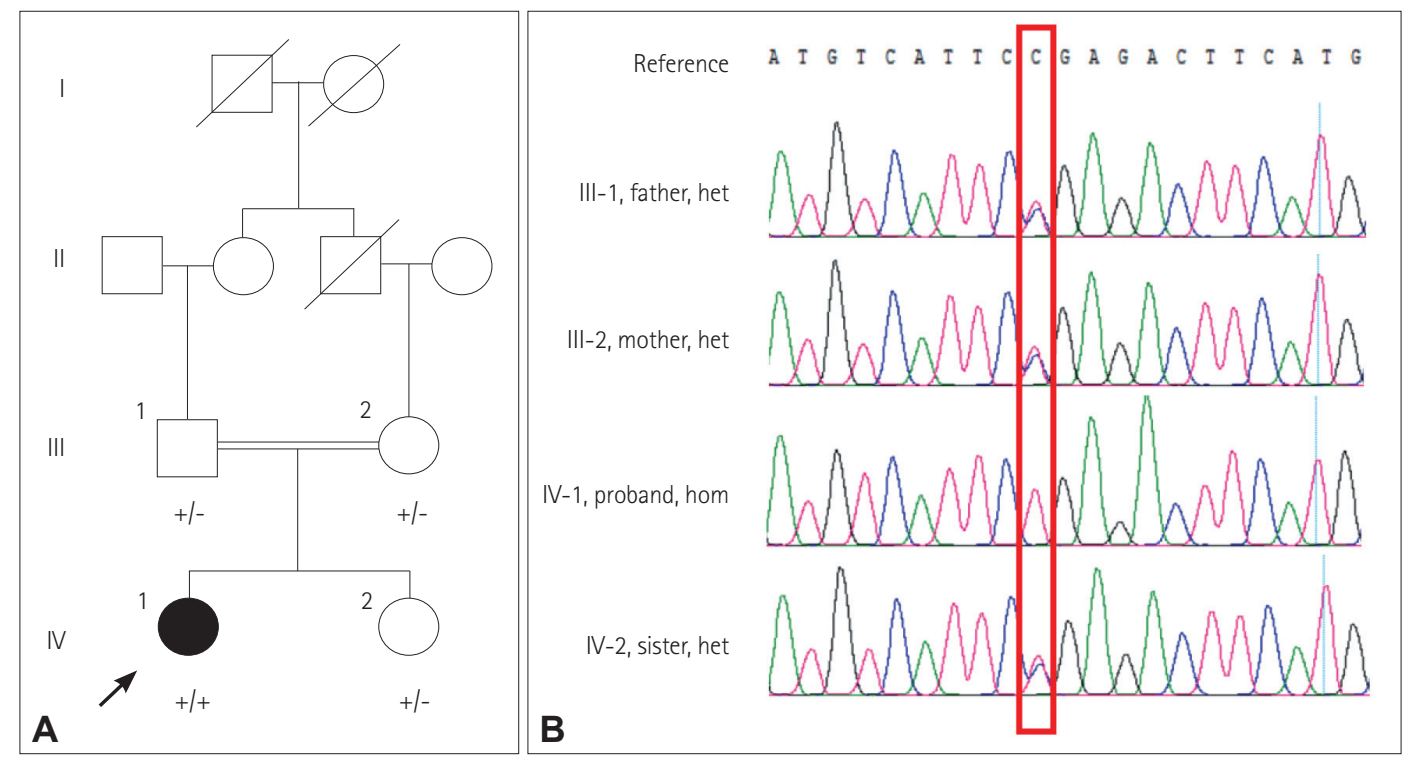

Fig. 1. The family tree. A: Black and white symbols indicate affected and healthy subjects, respectively. The arrow indicates the proband. Electropherograms of the variant c.1015C>T in CAPN1. B: The mutant nucleotide is boxed. het: heterozygous, hom: homozygous.

population.

\section{Conflicts of Interest}

The authors have no potential conflicts of interest to disclose.

\section{Acknowledgements}

The study was supported by the Science and Technology Department of Zhejiang Province (2019C03017), and the National Natural Science Foundation of China (No. 81870895, No. 81600850, No. 81571089 and No. 81371266 and No. 31671301).

We thank the patient and her parents and sister for their participation in this study.

\section{REFERENCES}

1. De Souza PVS, De Rezende Pinto WBV, De Rezende Batistella GN,
Bortholin T, Oliveira ASB. Hereditary spastic paraplegia: clinical and genetic hallmarks. Cerebellum 2017;16:525-551.

2. Gan-Or Z, Bouslam N, Birouk N, Lissouba A, Chambers DB, Vérièpe $\mathrm{J}$, et al. Mutations in CAPN1 cause autosomal-recessive hereditary spastic paraplegia. Am J Hum Genet 2016;98:1038-1046.

3. Wang Y, Hersheson J, Lopez D, Hammer M, Liu Y, Lee KH, et al. Defects in the CAPN1 gene result in alterations in cerebellar development and cerebellar ataxia in mice and humans. Cell Rep 2016;16:79-91.

4. Simonson L, Baudry M, Siman R, Lynch G. Regional distribution of soluble calcium activated proteinase activity in neonatal and adult rat brain. Brain Res 1985;327:153-159.

5. Forman OP, De Risio L, Mellersh CS. Missense mutation in CAPN1 is associated with spinocerebellar ataxia in the Parson Russell Terrier dog breed. PLoS One 2013;8:e64627. 